Modelling of congenital nystagmus waveforms produced by saccadic system abnormalities

Broomhead, D. S. and Clement, R. A. and Muldoon, M. R. and Whittle, J. P. and Scallan, C. and Abadi, R. V.

2000

MIMS EPrint: 2006.62

Manchester Institute for Mathematical Sciences

School of Mathematics

The University of Manchester

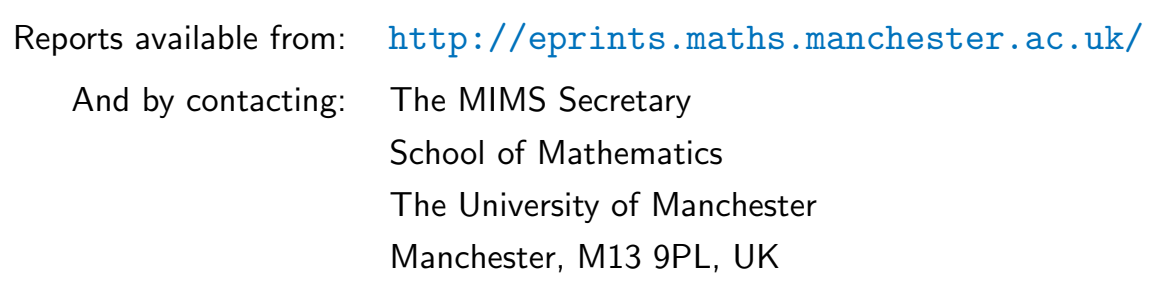




\title{
Modelling of congenital nystagmus waveforms produced by saccadic system abnormalities
}

\author{
David S. Broomhead ${ }^{1}$, Richard A. Clement ${ }^{2}$, Mark R. Muldoon ${ }^{1,3}$, Jonathan P. Whittle ${ }^{4}$, \\ Columba Scallan ${ }^{3}$ and Richard V. Abadi ${ }^{3}$ \\ 1 Department of Mathematics, UMIST, P.O. Box 88, Manchester M60 1QD, U.K. \\ 2 Defense Evaluation and Research Agency, Fort Halstead, Sevenoaks, Kent, TN14 7BP, U.K. \\ 3 Department of Optometry \& Neuroscience, UMIST, P.O. Box 88, Manchester M60 1QD, U.K. \\ 4 The University of Sheffield, Department of Ophthalmology and Orthoptics, Glossop Road, Sheffield S10 2JF, U.K.
}

Received: 12 May 1999 / Accepted in revised form: 19 November 1999

\begin{abstract}
Models of the mechanisms of normal eye movements are typically described in terms of the block diagrams which are used in control theory. An alternative approach to understanding the mechanisms of normal eye movements involves describing the eye movement behaviour in terms of smooth changes in state variables. The latter approach captures the burst cell firing against motor error (difference between target gaze angle and current gaze angle) phase plane behaviour which is found experimentally and facilitates the modelling of variations in burst cell behaviour. A novel explanation of several types of congenital nystagmus waveforms is given in terms of a saccadic termination abnormality.
\end{abstract}

\section{Introduction}

Normal fixation eye movements are driven by a combination of a burst of neural firing, which drives the eye muscle plant during the fast phase of the eye movement, and a tonic level of firing, which holds the eye in place during fixation (Robinson, 1964). The rate of firing of the burst cells is a nonlinear, saturating function of the dynamic motor error, which is the difference between the required eye position and the current eye position with respect to the orbit (Van Gisbergen et al. 1981). The accuracy of the fast phase eye movements, even under the effects of tranquillizers, shows that the burst signal is under feedback control (Jürgens et al. 1981).

Two alternative feedback models have been proposed: one based on estimated eye position; and one on estimated eye displacement (Zee et al. 1976, Van Gisbergen et al. 1981). The former-shown in Figure 1aassumes the burst cell firing pattern carries a velocity signal which is integrated to give a position signal. The

Send offprint requests to: David S. Broomhead dynamic motor error, $e$, is given by $e=g^{\star}-n$, the difference between the desired gaze angle $g^{\star}$ and an efference copy of eye position, $n$, taken from the neural integrator. The alternative model is based upon a resettable integrator which produces an estimate of the current eye displacement,s. In this case (Figure 1b), dynamic motor error (also labelled $e$ ) which drives the firing of the burst cells is equal to $\Delta g-s$, the difference between the required eye displacement, $\Delta g$, and the estimate of current eye displacement.

No neurophysiological evidence has been found to support the existence of a neural correlate of $g^{\star}$. On the other hand, there is strong evidence from neurophysiological studies of the superior colliculus (Scudder 1988) for a signal related to changes in the target position $(\Delta g)$. Because of this, more recent models of the saccadic system have been based on the displacement feedback model rather than the position feedback model (Breznen and Gnadt 1997; Dominey et al. 1997; Moschovakis 1994).

The displacement feedback model of normal saccadic eye movements also provides a basis for explaining a variety of abnormal saccadic eye movements. It is believed that the high gain of the burst cells can make the pulse generator inherently unstable in the sense that if a sufficient delay is introduced into the loop, then oscillations will occur. During fixation, such oscillations are suppressed by pause cells which inhibit the burst cells, since it has been found that the pause cells cease firing during a saccade (Keller 1977). If the pause cells do not provide a large enough inhibitory signal to the burst cells, then small amplitude (0.1-0.5 degrees) high frequency (15-30 $\mathrm{Hz}$ ) oscillations, referred to as microsaccadic flutter, can occur. If, in addition, the internal delay is increased to more than $20 \mathrm{~ms}$, then larger amplitude saccadic flutter is found (Ashe et al. 1991).

These explanations imply that microsaccadic and saccadic flutter are dynamical diseases. These have been 


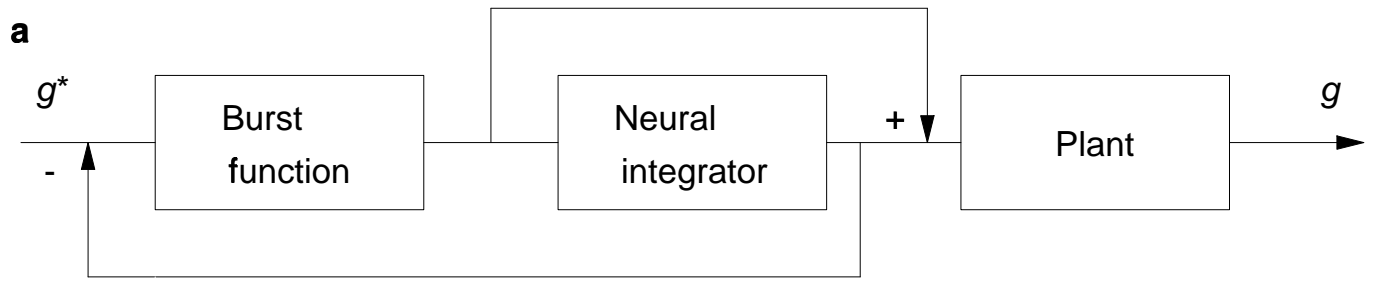

Estimate of eye position ( $\mathbf{n})$

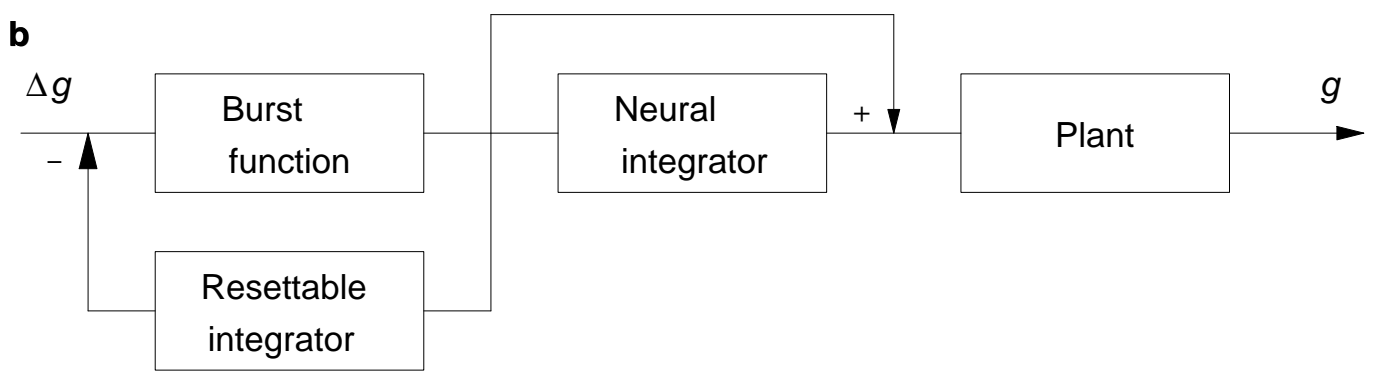

Estimate of eye displacement $(s)$

Fig. 1 Local feedback models of the saccadic system. The gaze angle with respect to the head is denoted by $g$, the angle to the target by $g^{\star}$ and the initial difference between the gaze angle of the eye and the target direction by $\Delta g$. (a) The position feedback model. A position signal $n$ is fed back from the neural integrator to generate the motor error. (b) The displacement feedback model. A copy of the output from the burst cells is integrated to obtain a current eye displacement signal $s$.

characterised as conditions which occur when the components of the system are still functioning, but are operating outside their normal range (Glass and Mackey 1979). In order to understand such conditions, it is appropriate to concentrate on the behaviour of the system, because structurally, the system may appear to be normal. The behaviour of a system can be analysed by application of nonlinear dynamics techniques, which involve describing the system by a set of coupled first order differential equations, and then analysing the geometric behaviour of the state variables in a multi-dimensional state space (Kaplan and Glass 1995, Wilson 1999).

The nonlinear dynamics approach is particularly suited to describing the behaviour of burst cells. The firing patterns of burst cells show characteristic trajectories when plotted in the burst cell firing against motor error phase plane. An advantage of this type of description of the behaviour of the burst cells is that it facilitates investigation of the effects of variations in the burst cell characteristics, as are found experimentally (Van Gisbergen et al. 1981). Using this method of analysis we find that burst cell firing can also be responsible for oscillations which have both a slow phase and a fast phase, as in the case of congenital nystagmus. The main results of this paper concern the properties of a bilateral version of a model of the saccadic system which predicts a number of congenital nystagmus waveforms when the burst cell characteristics are altered.

\section{A nonlinear dynamics model}

Both the plant and neural integrator can be treated as linear systems, and the only modification to the existing control theory models (Optican and Zee 1984, Van Gisbergen et al. 1981 and Van Opstal et al. 1985) which is needed, is to turn the description of the plant and neural integrator subsystem into a set of three first order differential equations, so that their description is compatible with the rest of the nonlinear dynamics model. A working description of the muscle plant is that it acts like a second order linear system with a slow time constant $T_{1}$ equal to 0.15 seconds and a fast time constant $T_{2}$ equal to 0.012 seconds (Optican and Zee 1984; Van Opstal et al 1985). The rate of change of the gaze angle, $g$, and eye velocity, $v$, are described by the pair of differential equations:

$$
\begin{aligned}
\frac{d g}{d t}=v & \\
\frac{d v}{d t}=-\left(\frac{1}{T_{1}}+\right. & \left.\frac{1}{T_{2}}\right) v \\
& +\frac{\left(-g+n+\left(T_{1}+T_{2}\right) b\right)}{T_{1} T_{2}}
\end{aligned}
$$


where $b$ is the input to the muscle plant from the burst cells and $n$ is the input from the neural integrator.

Similarly, the behaviour of the neural integrator can be modelled by a first order low pass filter with a time constant $T_{N}$, which is equal to 25 seconds in the normal oculomotor system (Optican and Zee 1984). This integrator can be described by a single equation:

$$
\frac{d n}{d t}=\frac{-n}{T_{N}}+b
$$

The qualitative behaviour of the burst cells associated with a saccade is similar to that of a heartbeat or nerve impulse in that it includes: 1) a stable equilbrium, 2) a threshold for triggering an action and 3) a return to equilibrium. The simplest system of differential equations which shows this type of behaviour is based on a cubic function (Zeeman 1972):

$$
\begin{aligned}
\epsilon \frac{d b}{d t} & =-b^{3}-b-e \\
\frac{d e}{d t} & =b
\end{aligned}
$$

where, as above, $b$ is the firing rate of the burst cells, $e$ is the dynamic motor error, and $\epsilon$ is a small positive number. In effect, whenever the dynamic motor error is nonzero, the state of the system relaxes rapidly onto the curve, $d b / d t=0$. The system then evolves according to (4). The result of this is that $b$ and $e$ decrease to zero along this curve. The curve $d b / d t=0$ is referred to as the nullcline of the burst cell equation (Kaplan and Glass 1995, Wilson 1999). The role of the multiplier $\epsilon$ is to constrain the dynamics of the burst cells to the burst cell firing rate nullcline. As $\epsilon$ is made smaller the system moves more and more rapidly onto the nullcline. The effect of altering the value of $\epsilon$ on the saccadic waveform will be explored in the next section.

A quantitative description of the burst cell activity must match the experimental findings which show that during a saccade, the firing level changes almost instantaneously from zero to lie on an exponential curve, and then follows this curve as the motor error decreases (Van Gisbergen et al. 1981). Hence the simple cubic function in the burst cell equation was replaced by an exponential function, the form of which was taken from previous descriptions of the relationship between motor error and burst cell firing rate (Van Gisbergen et al. 1981, Optican and Zee 1984). The parameters of the function were selected empirically so that durations and peak velocities of the modelled saccades followed the main sequence (Bahill et al. 1975). The firing rate of the burst cells can then be described by the equation:

$$
\epsilon \frac{d b}{d t}=-b+f\left(g^{\star}-n\right)
$$

where

$$
f(x)=\left\{\begin{aligned}
-800\left(1-e^{-|x| / 6}\right) & \text { if } x<0 \\
800\left(1-e^{-|x| / 6}\right) & \text { if } x \geq 0
\end{aligned}\right.
$$

In the position feedback model the motor error is given by the difference between the target gaze direction in head-based coordinates $g^{\star}$, and an estimate of current eye position in head-based coordinates $n$, which is obtained from the output of the neural integrator. So it is the combination of equations (3) and (5a) which determine the behaviour of the saccadic pulse generator, in the position feedback model. The displacement feedback model requires an additional equation in order to incorporate the resettable displacement integrator $s$, so the equations that determine the behaviour of the burst generator in the displacement feedback model are:

$$
\begin{aligned}
\epsilon \frac{d b}{d t} & =-b+f(\Delta g-s) \\
\frac{d s}{d t} & =b
\end{aligned}
$$

In the displacement feedback model, at the end of a normal saccade the displacement integrator is reset-a process not represented in (6). In the model of congenital nystagmus introduced in the next section the burst cell firing rate never falls to zero, so there is no indicator of the end of the saccade, and the integrator would not be expected to be reset.

In both types of feedback model, the two equations governing the activity of the burst cells are coupled in a feedback loop, so that, if the slope of the burst cell activity against motor error curve is reduced by a drug, then the pulse of innervation will be lengthened by the appropriate amount to ensure the accuracy of the saccade, as is found experimentally (Jürgens et al., 1981). The pulse activity of a saccade can be initiated by setting $g^{\star}$ equal to the required gaze direction with respect to the head in the position feedback model, and $\Delta g$ equal to the initial retinal error in the displacement feedback model. The solutions to these equations will then predict the time courses of the state variables during a saccade as shown in Figure 2. The mechanism of the pulse generator has been made clear in this figure by marking on the state space of the generator, vectors which show the state changes which are determined by (5b) and (6). If the pulse generator starts in a state at the tail of an arrow, then as time passes it will change to a state in the direction of the head of the arrow. Making the value of the parameter $\epsilon$ smaller will alter the lengths and directions of the vectors so that the trajectory associated with a single saccade is forced more directly onto the burst cell equation nullcline, and is forced to follow the nullcline more closely.

\section{A bilateral model of the saccadic system}

Individual horizontal burst cells fire maximally when the eye makes a saccade in a particular direction. So, if a cell fires maximally just before and during a saccade to the right, then it will be quiescent during a saccade to the 

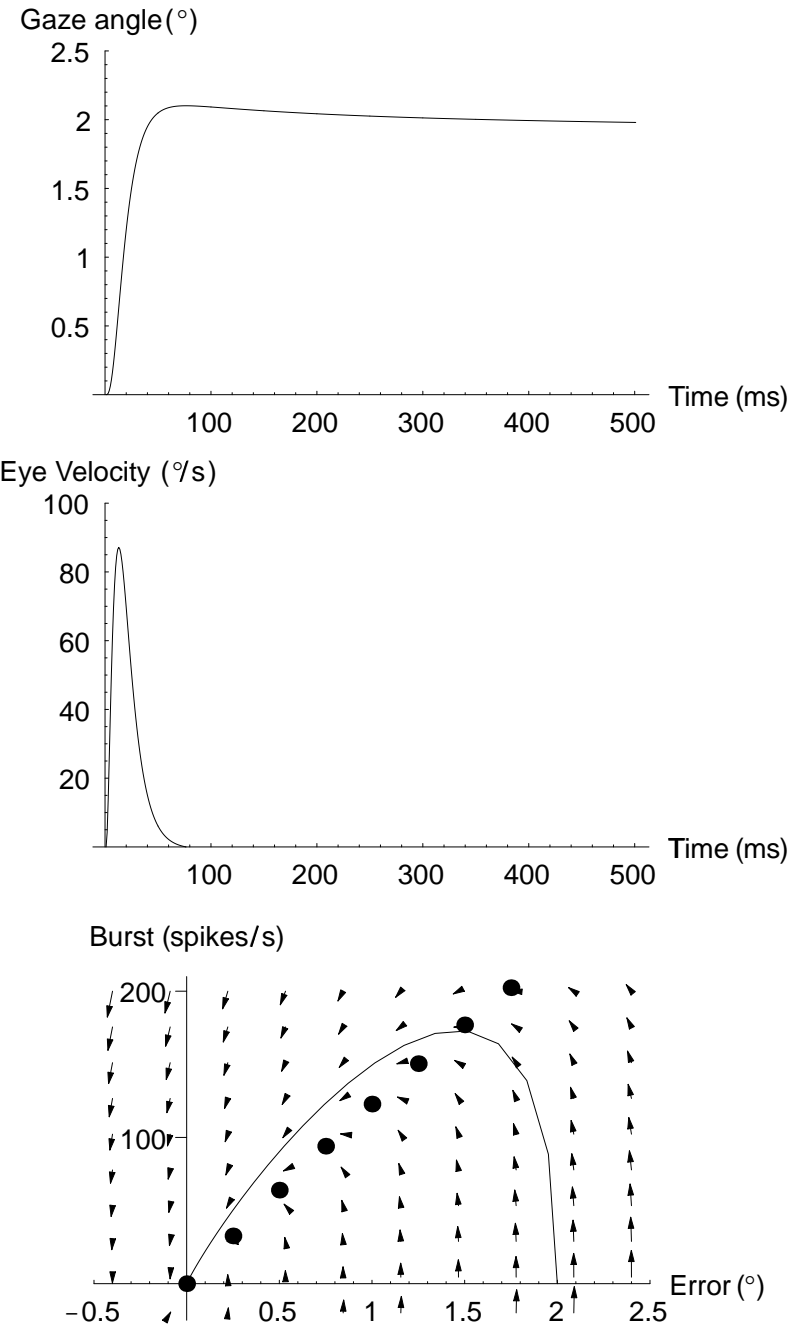

Fig. 2 The upper figures show the time course of the eye position and velocity for a 2 degree saccade generated by the model equations (5b) and (6) with $\epsilon=0.002$. The lowest figure is a phase plane portrait of the neural pulse generator. The motor error signal is plotted along the horizontal axis and the burst cell activity is plotted along the vertical axis. The arrows show how any state of the pulse generator will move to the next state. The dots mark the nullcline of the burst cell equation and the continuous curve marks the trajectory associated with the eye movement plotted in the upper figure.

left. The direction of eye movement for which a burst cell fires maximally is referred to as its 'on' direction, and the opposite direction is referred to as its 'off' direction. Although this description is approximately correct, it does not include the small responses associated with eye movements in the off direction which are found experimentally (Van Gisbergen et al. 1981).

A bilateral version of the displacement feedback model which more accurately reflects the experimentally determined burst cell functions can be made by incorporating two further features. Firstly, the experimentally found off response of burst cells can be included and secondly, reciprocal inhibition between the left and right bursters is needed to ensure that co-contraction does not occur at the end of a saccade. In order to ensure that either the left or the right bursters dominate, it is appropriate to use nonlinear rather than linear reciprocal inhibition between left and right bursters. The simplest modification to the displacement feedback model which incorporates these two features, involves replacing equation (5b), which describes the firing of the burst cells, by a pair of symmetrical equations which describe the firing of separate populations of left $l$ and right $r$ bursting cells. In this version the overall burst cell signal $b$ is given by the difference between the right and left populations, i.e. $b=r-l$. Then:

$$
\begin{aligned}
\frac{d b}{d t}=\frac{d r}{d t}-\frac{d l}{d t} \\
\epsilon \frac{d l}{d t}=-l-0.05 l r^{2}+ \\
\quad f(-(\Delta g-s))+h(-(\Delta g-s)) \\
\epsilon \frac{d r}{d t}=-r-0.05 r l^{2}+f(\Delta g-s)+h(\Delta g-s)
\end{aligned}
$$

where

$$
\begin{aligned}
& f(x)=\left\{\begin{array}{cl}
0 & \text { if } x \leq 0 \\
800\left(1-e^{-x / 6}\right) & \text { if } x>0
\end{array}\right. \\
& \text { and } \\
& \qquad h(x)=\left\{\begin{array}{cc}
-200 \alpha\left(\frac{x}{1.5 \beta}\right) e^{x /(1.5 \beta)} & \text { if } x<0 \\
0 & \text { if } x \geq 0
\end{array}\right.
\end{aligned}
$$

The function $f$ now describes the firing of a burst cell in its on direction only, while $h$ is a function which describes the firing of a burst cell in its off direction only. The forms of both of these functions were based on experimental measurements (Van Gisbergen et al. 1981). This model makes it easy to investigate the consequences of alterations in the form of the off responses of burst cells, which show a greater variety of relationships with the motor error than do the on responses (Van Gisbergen et al. 1981). The constant $\alpha$ alters the maximum amplitude of the off response and the constant $\beta$ alters the range of motor error over which the off response operates. Ideally, an off function $h$ in which the maximum amplitude and effective range could be altered completely independently would be most revealing. However, with the simple $h$ function specified in $(5 \mathrm{c})$, any change in $\alpha$ alters the maximum amplitude of the off response, but also, to a lesser extent, the range of motor error over which the off response operates. This is also the case for $\beta$. Consequently, some modifications to other parameters may be needed to ensure that the waveforms remain realistic.

The time course and velocity of a normal 2 degree saccade predicted by the model is plotted in Figure 3a. Because there are now two burst cell equations the state space is three-dimensional, and the nullclines of each equation are surfaces. However, the equations are such as to force the state of the pulse generator onto the intersection of the left and right burst cell nullclines, which 

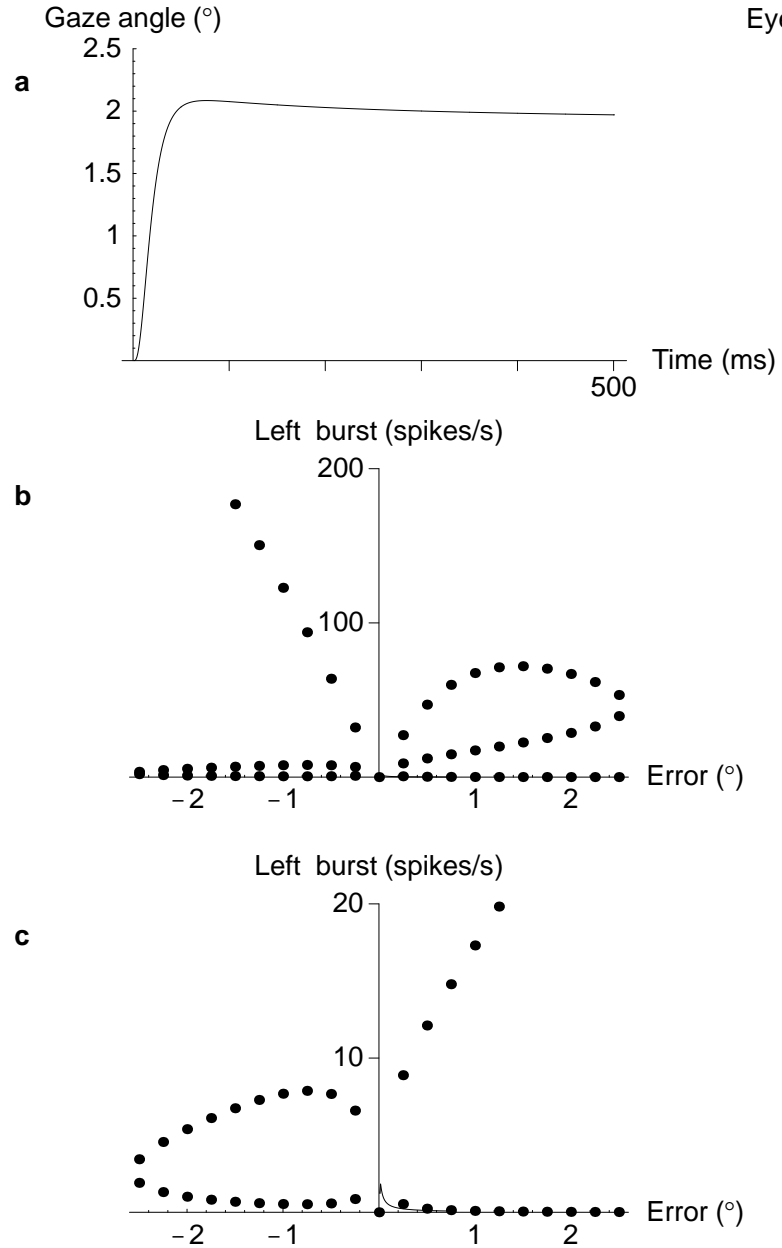

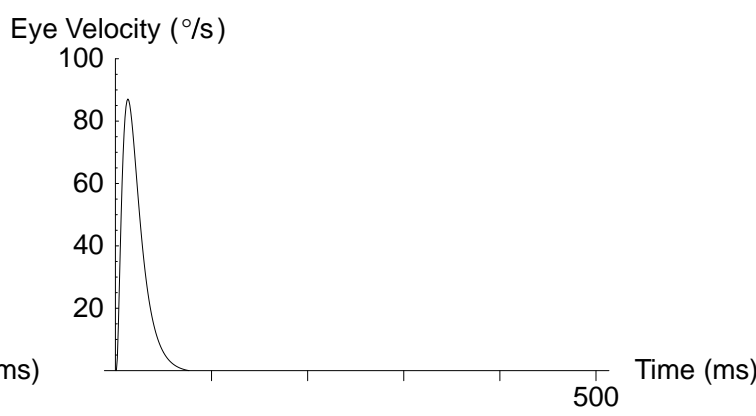

Right burst (spikes/s)

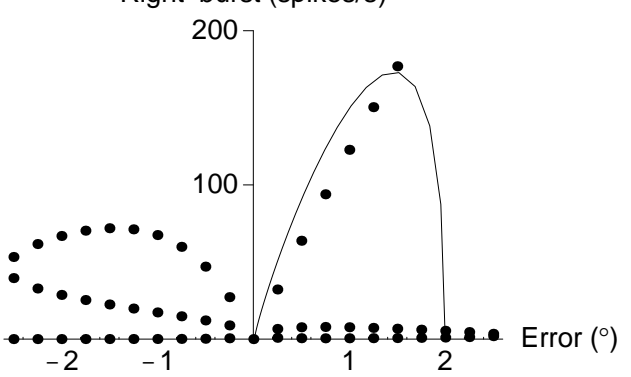

Right burst (spikes/s)

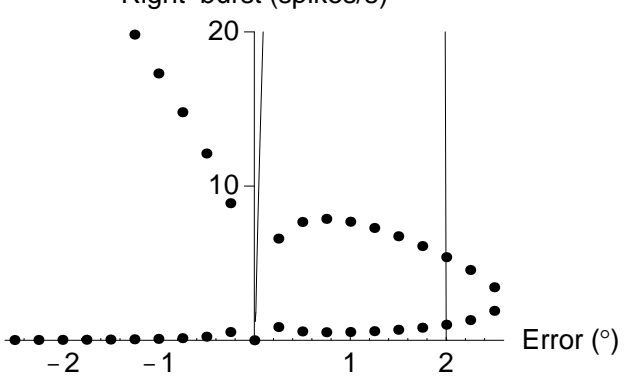

Fig. 3 Time course and phase portraits of burst cell activity for a 2 degree saccade of the bilateral model; $\alpha=1, \beta=1$ and $\epsilon$ $=0.002$. (a) Time course and velocity profile of the eye movement; (b) phase portraits of the right and left burst cell activity; (c) expanded portion of the phase portrait in (b). See text for detailed discussion.

is a curve in three-dimensional space. To preserve consistentency with the simpler model, separate plots of the burst cell activity against motor error have been made for the left and right burst cells in Figure 3b. These are projections into two dimensions of the trajectories in the three-dimensional state space.

The curve onto which the state of the pulse generator is forced is again shown by a dotted line. The form of this curve can be understood by considering the points on the curve associated with a given value of motor error $e$. When $e$ is fixed, the burst cell equations are equivalent to those of a pair of mutually inhibitory nonlinear neurons, which typically have three equilibrium points, two of which are stable and one which is unstable (Kaplan and Glass 1995, Schamma 1989). For example, if $e$ $=1$ it can be seen, in Figures $3 \mathrm{~b}$ and $3 \mathrm{c}$ (the latter is a magnified version of the former) that there are three firing levels of the right burst cell at $0.5,7.7$, and 122.8 spikes/sec. Trajectories are repelled from the portion of the dotted curve passing through the point at $(e, b)=(1$, $7.7)$, but attracted to the portions of the curve passing through $(1,0.5)$ and $(1,122.8)$.
With normal saccades, the trajectory of the burst cell for which the movement is in its off direction, which in this case is the left burst cell, passes along the set of stable points close to the zero firing level. The very small off response, which occurs when $e$ is small, is visible in Figure 3c. If, for some reason, the trajectory switches to pass along the attracting portion of the dotted curve farthest from the zero firing level, then the model produces eye movement waveforms which are typical of saccadic eye movement disorders. Note that this possibility is inherent in the dynamical structure which is present even in the normal eye movement portrayed in Figure 3.

For example, if the range of motor errors over which the off component of a burst cell operates is reduced, then microsaccadic oscillations occur. This is illustrated in Figure $4 \mathrm{a}$, in which the value of $\beta$ in the $h$ function has been changed to 0.333 , which corresponds to a range of motor error of 2 degrees, as opposed to the normal range of 5 degrees. With $\beta=2.333$, which corresponds to a range of 12 degrees, then nystagmus with pseudocycloid waveforms occurs, as in Figure 4b (Dell'Osso and Daroff 1975). This type of waveform typically occurs in 
a

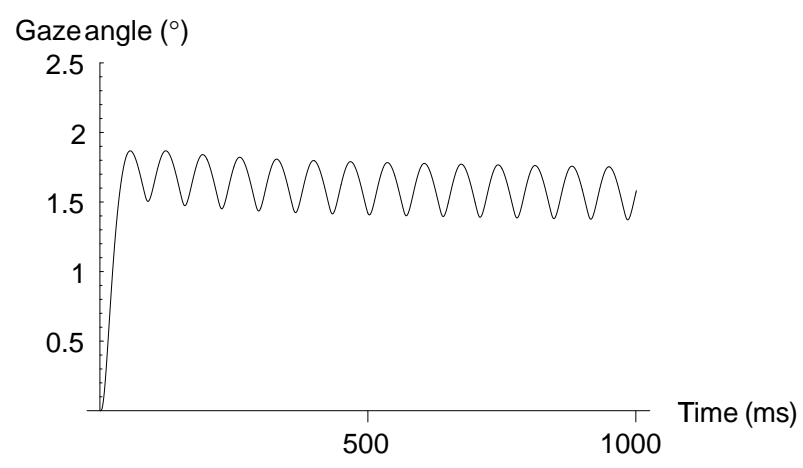

c

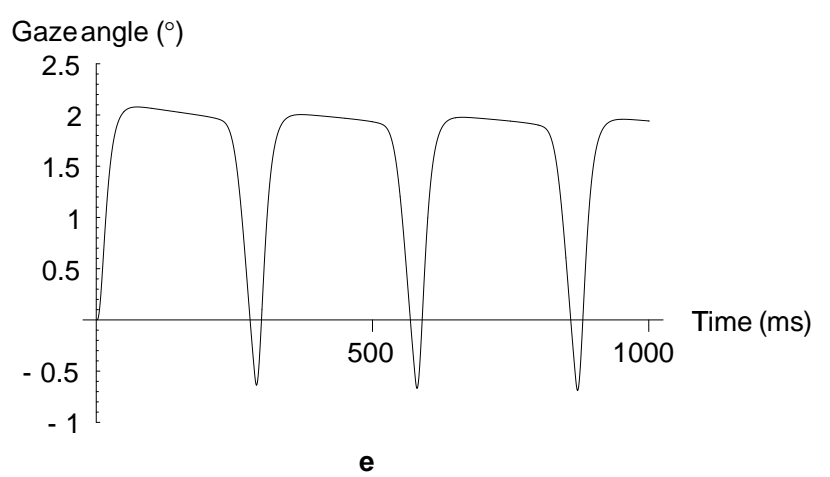

Gaze angle $\left(^{\circ}\right)$

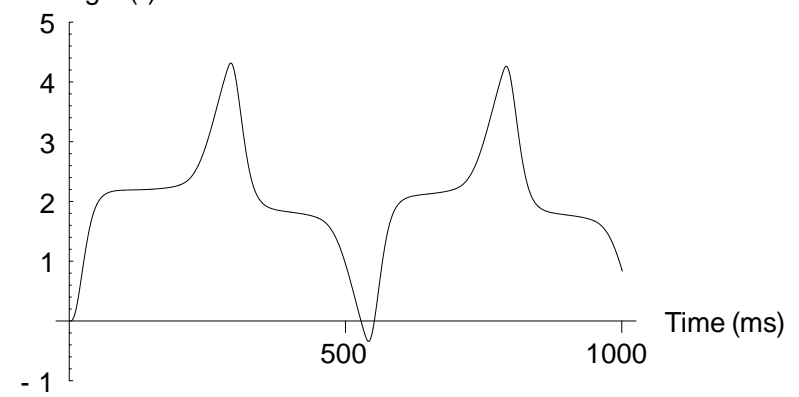

Gazeangle $\left(^{\circ}\right)$

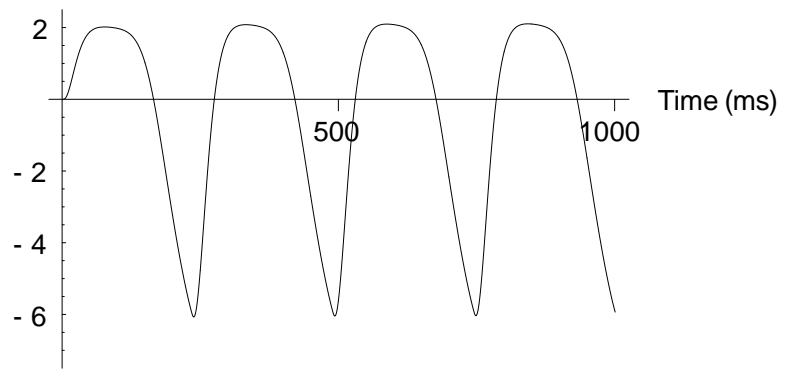

d

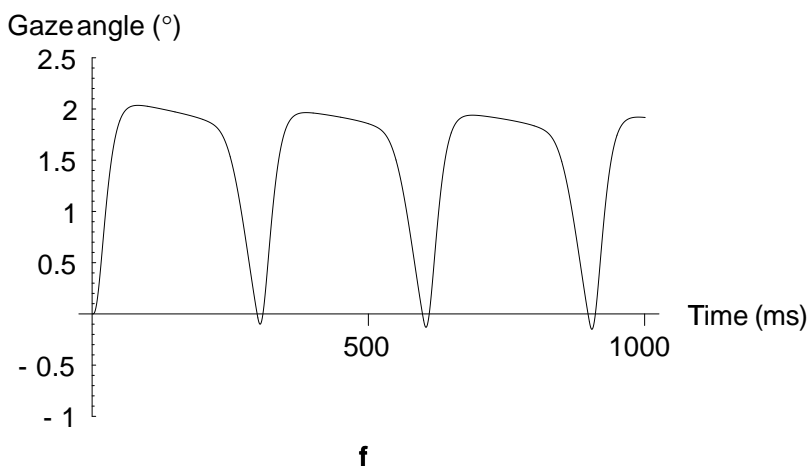

Gaze angle $\left(^{\circ}\right)$

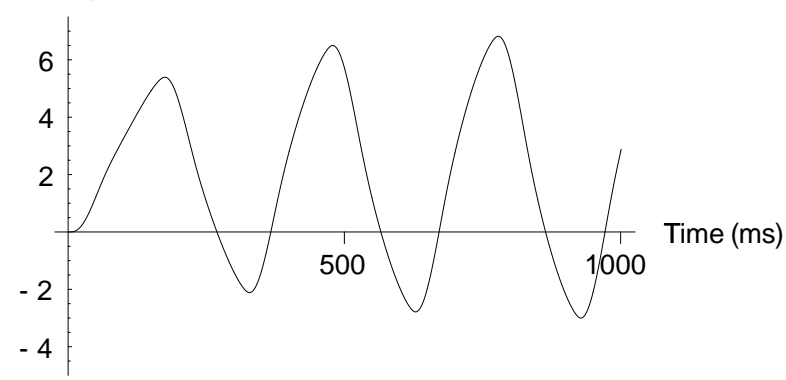

Fig. 4 Varieties of waveforms produced by changing the parameters of the model. (a) $\alpha=0.3, \beta=0.333$ and $\epsilon=0.002$ : microsacadic flutter. (b) $\alpha=1.35, \beta=2.333$ and $\epsilon=0.0035$ and congenital nystagmus on burst function: pseudo-cycloid waveforms. (c) $\alpha=1.05, \beta=1.0$ and $\epsilon=0.002$ : jerk nystagmus. (d) $\alpha=0.55, \beta=1.0$ and $\epsilon=0.0035$ and congenital nystagmus on burst function: jerk nystagmus. (e) $\alpha=0.55, \beta=1.0$ and $\epsilon=0.0065$ and congenital nystagmus on burst function: bias reversal. (f) $\alpha=0.55, \beta=1.0$ and $\epsilon=0.05$ and congenital nystagmus on burst function: pendular nystagmus.

cases of congenital nystagmus, in which the saccades are usually slower than normal (Abadi and Worfolk 1989). To make the waveforms realistic the on burst function $f$ was replaced by one appropriate for the slower saccades in congenital nystagmus:

$$
f(x)=\left\{\begin{array}{cc}
0 & \text { if } x \leq 0 \\
600\left(1-e^{-x / 9}\right) & \text { if } x>0
\end{array}\right.
$$

If the amplitude of the off response is increased then a jerk congenital nystagmus waveform appears, as shown in Figure 4c. Combining the off burst function appropriate for congenital nystagmus with suitably chosen values of $\alpha$ and $\epsilon$ makes the distinction between fast and slow phases is clearer, as can be seen by comparing Figure 4c with 4 d.

Figures 4d, 4e, 4f and Figure 5 illustrate the effect of the $\epsilon$ parameter, which determines how closely the trajectory of the burst cell firing follows the $d l / d t$ and $d r / d t=0$ curve. As $\epsilon$ increases, the manner in which the trajectory follows the dotted curve becomes increasingly sloppy, overshooting changes in direction of the curve, and the eye movements become larger and the transitions between the fast and slow phases become less sharp. In Figure 4e, it can be seen that with $\epsilon=0.0065$ 

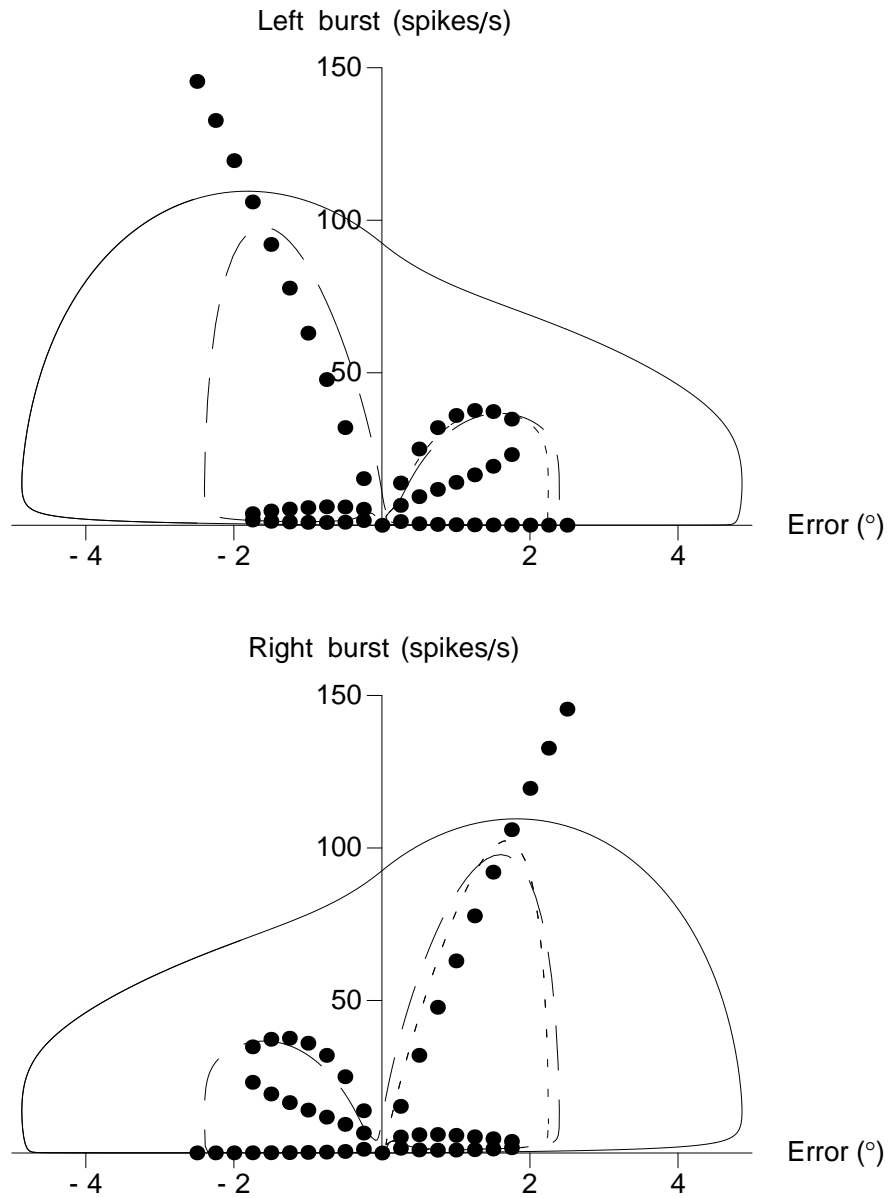

Fig. 5 Phase plane trajectories of the burst cell firing associated with the jerk nystagmus of Figure 4d (short dashes), the reversal of Figure 4e (long dashes) and the pendular nystagmus of $4 \mathrm{f}$ (continuous). The trajectory of the right burst cells proceeds anticlockwise during the course of the nystagmus, while that of the left burst cells proceeds in the opposite direction

the trajectory of left burst cell overshoots the origin and so successive cycles of the nystagmus alternate in phase. Figure $4 \mathrm{f}$ shows the effect of a further increase in $\epsilon$, which causes the eye movement to become slower and pendular, with no discernable transition between the fast and slow phases.

Because the bilateral model presented in this section provides an explanation of how congenital nystagmus waveforms could emerge as a consequence of anomalous off reponses by the burst cells, it also predicts that voluntary saccades cannot be made independently of the ongoing nystagmus. Although voluntary saccades in the directions of the quick phases will be normal, voluntary saccades in the direction of the slow phases will act to push the state of the burst generator back down the fold of the dotted curve. With large saccades the state will be pushed across the zero motor error line and will result in a normal saccade. But small saccades will fail to push the state across the zero motor error line and will simply result in an extended slow phase. The predictions are illustrated in Figure 6. Experimental studies provide evidence for this effect, in that saccades in the direction of the quick phase are normal, whereas saccades in the opposite direction to the quick phase can either result in a saccade or an extended slow phase (Bedell et al. 1987, Worfolk and Abadi 1991). However, although the extended slow phase is more common with small saccades, the occurrence of an extended slow phase is not as absolutely tied to saccade size as predicted by the model.

\section{Discussion}

Our starting hypothesis was that microsaccadic and saccadic flutter occur when the activity of the pause cells is abnormally low (Ashe et al. 1991). Clearly, a sequence of saccades with no interval between them cannot occur if the pause cells are inhibiting burst cell activity normally at the end of each saccade. Next, a more realistic model of burst cell activity was introduced, and it was found that changes in the parameters governing the off direction responses of the burst cells, which are naturally more variable, gave rise to the types of waveforms typically found in congenital nystagmus. So, by applying a more accurate description of experimentally determined burst cell activity in the context of the current hypothesis about saccadic system disorders, we arrived at a new hypothesis: that a disorder of the saccadic termination mechanism underlies congenital nystagmus.

The explanation of the generation of congenital nystagmus developed within the framework of the reciprocal inhibition model of the organisation of the burst cells rests on an abnormality of the relationship between burst cell activity and motor error. The viability of such an explanation cannot be properly assessed without greater understanding of how the normal relationship between burst cell activity and motor error develops, and our model of nystagmus highlights this problem. It may be envisaged that early in life many burst cells receive excitatory connections so that they fire in both on and off directions of saccades. After a while, a process of competitive learning, if successful, results in burst cells which are tuned to fire in predominantly one direction. The question then arises, as to why in some cases the development process is not successful.

The most comprehensive previous explanation of the variety of congenital nystagmus waveforms was provided by Optican and Zee (1984). In their model the time constant of the neural integrator is lengthened by a velocity feedback signal, and when the sign of the feedback signal is reversed, the small postsaccadic drift velocities are amplified by the unstable velocity feedback loop, leading to exponentially growing slow phases. There are two main differences which arise from explaining congenital nystagmus waveforms in terms of a saccadic termination malfunction as opposed to a malfunction of the neural integrator. 


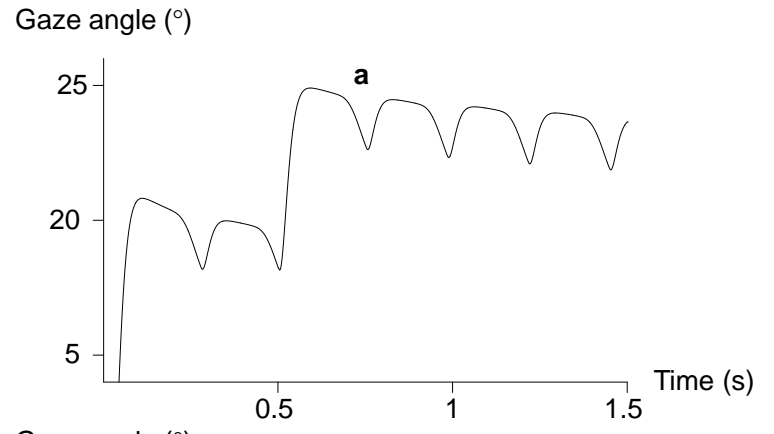

Gaze angle $\left(^{\circ}\right)$

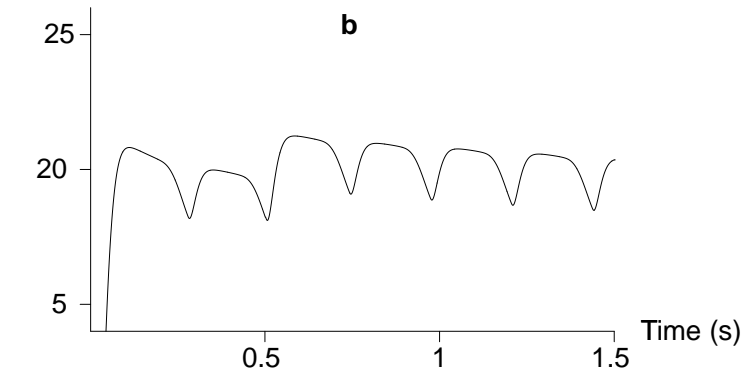

Gaze angle $\left({ }^{\circ}\right)$

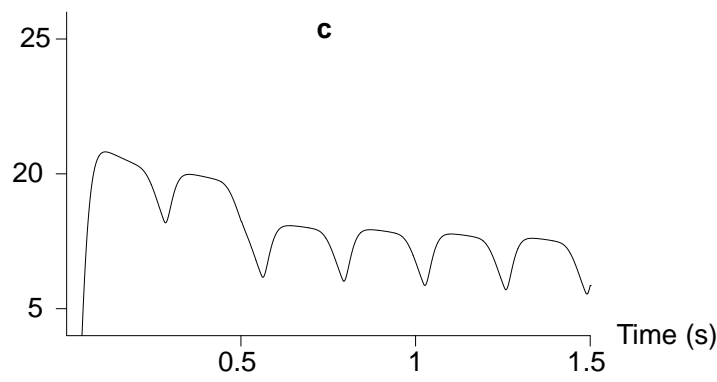

Gaze angle $\left({ }^{\circ}\right)$

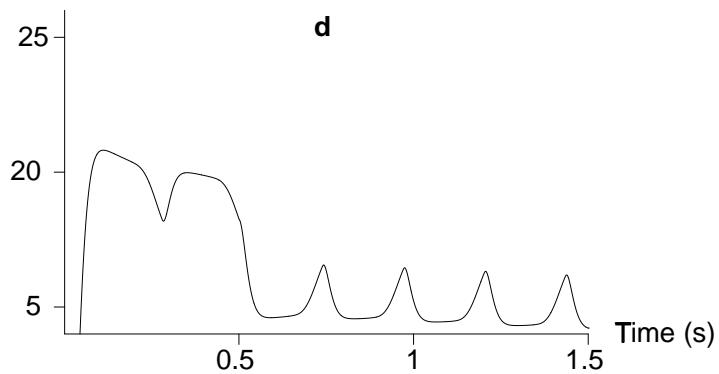

Fig. 6 Predicted interaction of a voluntary saccade with an ongoing congenital nystagmus waveform. (a) A 5 degree voluntary saccade in the direction of the fast phase. (b) A 1.5 degree voluntary saccade in the direction of the fast phase. (c) A 1.5 degree voluntary saccade in the direction opposite to the fast phase. (d) A 5 degree voluntary saccade in the direction opposite to the fast phase.

Firstly, in order for the pulse of innervation to appear normal despite a malfunctioning neural integrator, the burst cells are required to function normally. By comparison, in our saccadic termination abnormality model, abnormal burst cell activity can explain both microsaccadic oscillations, and the interactions between congen- ital nystagmus and a voluntary saccade, as well as the occurrence of certain congenital nystagmus waveforms.

Secondly, the waveform associated with the neural integrator malfunction model will be gaze angle dependent, which is not the case for models based on malfunctions of the saccadic or pursuit systems (Harris 1995). A similar position dependence could be incorporated in the saccadic termination model by making the reciprocal inhibition position dependent. As it stands, the bilateral model described in the Section 3 provides an explanation for waveforms seen near the primary position, but needs to be extended to explain congenital nystagmus seen in eccentric gaze positions. For example, in Figure 6d, the voluntary saccade in the opposite direction to the quick phase results in a reversal of the direction of the nystagmus which is not found in experimental recordings of the nystagmus.

The two models differ most in their predictions as to the occurrence of pendular nystagmus. The Optican and Zee (1984) model only generates small amplitude sinusoidal pendular nystagmus close to the null region, whereas our saccadic termination abnormality model only produces large angle sinusoidal pendular nystagmus! In part this difference arises because Optican and Zee required the pendular nystagmus to be purely sinusoidal, which is rarely the case in practice (Reinecke et al. 1988). Indeed, the most problematical waveform for the saccadic termination abnormality model to explain is that of pendular nystagmus with foveating saccades. To model the pendular nystagmus the factor $\epsilon$ must be large, but to model the foveating saccades, the factor $\epsilon$ must be small. Explanation of changes in nystagmus waveforms in terms of changes in the the factor $\epsilon$ are only viable when there are changes in the entire waveform, and there are at least two situations where this occurs. Firstly, when a subject becomes sleepy, the waveform becomes slower and more rounded (Abadi and Dickinson 1986), so that in this case the factor $\epsilon$ could be taken to reflect the state of arousal of the subject. Secondly, congenital nystagmus in babies often appears as a slow, large amplitude pendular-like nystagmus which often develops later in life into a jerk waveform nystagmus. In this case the development of the oculomotor system could be modelled by steadily reducing the factor $\epsilon$.

The main failing of all the quantitative models of congenital nystagmus that have been developed so far, including the one presented here, is that they all settle down into a periodic waveform (Harris 1995, Optican and Zee 1984, Tusa et al. 1992). Whilst incomplete, models which predict periodic waveforms are consistent with nystagmus data in the foveation region (Abadi et al. 1997). What is needed is a quantitative characterisation of the additional mechanisms which operate outside the foveation region. 


\section{References}

1. Abadi RV, Broomhead DS, Clement RA, Whittle JP and Worfolk R (1997) Dynamical systems analysis: a new method of analysing congenital nystagmus waveforms. Exp Brain Res 117: 355-361.

2. Abadi RV and Dickinson CM (1986) Waveform characteristics in congenital nystagmus. Doc Ophthalmol 64: 153-167.

3. Abadi RV and Worfolk R (1989) Retinal slip velocities in congenital nystagmus. Vision Res, 29: 195-205.

4. Ashe J, Hain TC, Zee DS and Schatz NJ (1991) Microsaccadic flutter. Brain 114: 461-472.

5. Bahill AT, Clark MR and Stark L (1975) The main sequence, a tool for studying eye movements. Math Biosci 24: 191-204.

6. Bedell HE, Abplanalp PL and McGuire CA (1987) Oculomotor response to target displacement by patients with congenital idiopathic nystagmus and nystagmus associated with albinism. Clin Vis Sci 2:21-31.

7. Breznen B. and Gnadt JW (1997) Analysis of the step response of the saccadic feedback: computational models. Exp Brain Res 117:181-191.

8. Dell'Osso LF and Daroff RB (1975) Congenital nystagmus waveforms and foveation strategy. Doc Opthal 39: 155-182.

9. Dominey PF, Schlag J, Schlag-Rey M, Arbib MA (1997) Colliding saccades evoked by frontal eye field stimulation: artifact or evidence for an oculomotor compensatory mechanism underlying double-step saccades. Biol Cybern 76: 41-52.

10. Gancarz G and Grossberg S (1998) A neural model of the saccadic generator in the reticular formation. Neural Networks 11: 1159-1174.

11. Glass L and Mackey MC (1979) Pathological conditions resulting from instabilities in physiological control systems. Ann. N.Y.Acad.Sci. 316, 214-35.

12. Harris CM (1995) Problems in modelling congenital nystagmus: Towards a new model. In: Findlay JM, Walker R and Kentridge RW (eds) Eye Movement Research Mechanisms, Processes and Applications. North-Holland, Amsterdam pp 239-253.

13. Jürgens R, Becker W and Kornhuber HH (1981) Natural and drug-induced variation of velocity and duration of human saccadic eye movements: evidence for a control of the neural pulse generator by local feedback. Biol Cybern 39: 87-96.

14. Kaplan D and Glass L (1995) Understanding Nonlinear Dynamics. Springer-Verlag, New York.

15. Keller EL (1977) Control of saccadic eye movements by midline brainstem neurons. In: Baker $\mathrm{R}$ and Berthoz A (eds) Control of Gaze by Brainstem Neurons. Elsevier, North-Holland, Amsterdam pp. 327336.

16. Moschovakis AK (1994) Neural network simulations of the primate oculomotor system. Biol Cybern 70: 291-302.

17. Optican LM and Zee DS (1984) A hypothetical explanation of congenital nystagmus. Biol Cybern 50: 119-134.

18. Reinecke, R, Guo S, and Goldstein HP (1988) Waveform evolution in in infantile nystamus: an electrooculographic study of 35 cases. Binocular Vision 3: 191-202.
19. Robinson DA (1964) The mechanics of human saccadic eye movements. J.Physiol 174: 245-264.

20. Schamma S (1989) Spatial and temporal processing in central auditory networks. In: Koch C and Segev I (eds) Methods in Neuronal Modelling, MIT Press, Cambridge, Massachusetts. pp 247-289.

21. Scudder CA (1988) A new local feedback model of the saccadic burst generator. J Neurophysiol 59: 14551475 .

22. Tusa RJ, Zee DS, Hain TC and Simonsz HJ (1992) Voluntary control of congenital nystagmus. Clin Vis Sci 7: 195-210.

23. Van Gisbergen JAM, Robinson DA and Gielen S (1981) A quantitative analysis of generation of saccadic eye movements by burst neurons. J Neurophysiol, 45: 417-442.

24. Van Opstal AJ, Van Gisbergen JAM and Eggermont JJ (1985) Reconstruction of the neural control signal for saccades based on an inverse method. Vision Res 25: 789-801.

25. Wilson HR (1999) Spikes, decisions and actions. Oxford University Press, Inc., New York.

26. Worfolk R and Abadi RV (1991) Quick phase programming and saccadic re-orientation in congenital nystagmus. Vis Res 31: 1819-1830.

27. Zee DS, Optican LM, Cook JD, Robinson DA and Engel WK (1976) Slow saccades in spinocerebellar degeneration. Arch Neurol, 33: 343-351.

28. Zeeman EC (1972) Differential equations for the heartbeat and nerve impulse. In: C.H.Waddington (ed) Towards a Theoretical Biology, vol. 4, Edinburgh University Press. pp. 8-67. 